\title{
Intramedullary Nailing of Periarticular Fractures
}

\author{
Walter Virkus, Laurence Kempton, Anthony Sorkin, Greg Gaski
}

Introduction

Intramedullary nailing (IMN) has numerous advantages for fracture fixation including its potential for minimally invasive exposure, biologically friendly implant insertion, longer implants to span more complex fractures, and load sharing fixation to allow earlier weight bearing. These clinical advantages and recent improvements in implant design have generated interest in expanding the indications for IMN. As IMN is utilized for more metaphyseal and periarticular fractures, technique-related complications have been identified. Malreductions often occur because nails do not inherently align metaphyseal segments as they do with simple diaphyseal fractures. In this article, we will discuss indications for periarticular intramedullary nailing (PIMN), techniques to achieve optimal results, and supporting literature.

\section{Indications and Contraindications}

Recent advances in implant design include multiplanar interlocks clustered near the ends of nails, facilitating improved purchase in epiphyseal segments. Many modern implants have the ability to "lock" the interlocks to the nail, creating a fixed-angle construct and theoretically improving stability. In many cases, these implants provide similar stability to plate fixation. ${ }^{1}$ PIMN is particularly advantageous in patients at higher risk for wound complications such as those with diabetes, morbid obesity, peripheral vascular disease, thin skin, and compromised soft tissue (e.g. open fractures). ${ }^{2}$ Nails offer certain mechanical advantages over plates in spanning long segments of comminution and are thus an attractive option for periarticular fractures with

This is the author's manuscript of the article published in final edited form as:

Virkus, W., Kempton, L., Sorkin, A., \& Gaski, G. (2018). Intramedullary Nailing of Periarticular Fractures. Journal of the American Academy of Orthopaedic Surgeons, 26(18), 629-639. https://doi.org/10.5435/JAAOS-D-16-00849 
diaphyseal extension and segmental injuries. ${ }^{3,4}$ Fractures with intra articular extension can be treated with PIMN after anatomic articular reduction has been obtained, provided all supplemental plates and screws are kept outside of the planned nail trajectory. Proximal tibia fractures with separate tibial tuberosity fragments are poor candidates for PIMN as the nail causes a significant anteriorly directed deforming force. Periprosthetic distal femur fractures are usually amenable to retrograde nailing as most modern total knee (TKA) implants have an open box in the femoral component.

The amount of fixation required through the nail in the epiphyseal segment is a critical question without a data driven answer. The authors have generally observed successful outcomes if a minimum of 2 bicortical interlocks can be inserted into the epiphyseal segment. Results of early weightbearing after nailing of periarticular fractures are unknown. We are only aware of one study which commented on weightbearing in a periarticular nailing study, and those authors did not allow weightbearing until complete radiographic union [24]. We have unpublished data on 2 cohorts of elderly distal femur fractures treatetd with PIMN. One group was allow weightbearing as tolerated (WBAT), and the other protected weightbearing. There was no significant difference in fixation failures between the groups. Our typical practice is to allow non articular fractures to allow WBAT for nonarticular fractures, and protected weightbearing for articular fractures. Patient and injury factors such as obesity, comminution, and bone quality can impact this decision on a case by case basis.

General Periarticular Nailing Techniques

PIMN relies heavily on a variety of techniques intended to influence the reduction of the short segment during reaming and nail insertion. The first and most important technique is 
obtaining the appropriate starting point. Malreduction secondary to a poor starting point can often be corrected with one or more of the other techniques described in this section.

Obtaining and maintaining reduction throughout reaming, nail insertion, and interlocking is critical for successful PIMN. This can be achieved with reduction clamps placed percutaneously through stab incisions prior to reaming and nail placement. ${ }^{5}$ Bone hooks, cobb elevators, and co-linear reduction forceps can also be applied through limited incisions to effect reduction.

Another commonly applied technique is the placement of blocking drill bits or screws. ${ }^{6}$ These require a segment without comminution for bicortical stabilization. A rule of thumb is to place them in the concavity of an angular deformity. They are always placed in a location where the nail needs to be redirected. For example, a blocking screw placed posterior to the guidewire, will direct the nail anteriorly and avoid an apex-anterior deformity. Using drill bits (>4mm) rather than screws allows for quick positional adjustments without committing to a particular location. Care should be taken when reaming around drills bits as the spinning of the reamer can advance the drill bit through the bone into the soft tissue. When using drill bits as blocking devices, make sure that the nail is locked both proximally and distally prior to removal. If the intention is to definitively leave a blocking device in place after nail insertion, separate blocking screws should be placed prior to removal of blocking drill bits; the potential deforming forces seen by the drill bit may not allow reinsertion of the screw in the same bicortical path. The further away from the interlocks a blocking drill bit is positioned, the more likely it will need to be replaced by a blocking screw to maintain reduction

A fourth technique used in extreme nailing is the application of adjunctive plates through a small incision at the fracture site. ${ }^{7,8}$ The plates vary in size depending on the location and 
intended mode for use. Buttress fixation of metaphyseal shear fractures are best reduced with $3.5 \mathrm{~mm}$ recon or one-third tubular plates with bicortical screws out of the nail path. Minifragment plates with unicortical screws can help appose cortical surfaces to maintain reduction without interfering with nail placement.

\section{Distal Femur Fractures}

\section{Pearl 1: Positional reduction}

Retrograde nailing of distal femur fractures is performed supine on a radiolucent table. This position facilitates the use of strategically placed bumps or triangles in varying positions creating flexion or extension of the fracture to obtain reduction. ${ }^{5}$ A large periarticular clamp placed across the femoral condyles can provide a direct "handle" on the distal fragment, and manual traction through this clamp can provide additional assistance with reduction. Applying the clamp more anterior or posterior provides a flexion or extension moment to the distal femur to counteract sagittal plane deformity. Restoration of length and coronal plane alignment can be easily achieved with one hand on the clamp while reaming with the other hand. This "traction clamp" is particularly helpful in comminuted fractures for which direct clamping and blocking screws can be impossible.

Judging proper rotation is facilitated by assessing the rotational profile of the contralateral extremity prior to draping and then matching this during reaming and nail insertion. Alignment should be checked repeatedly with AP and lateral fluoroscopy during reaming and nail placement. Rotation and length should be rechecked both radiographically and clinically after one proximal locking drill bit is placed. 


\section{Pearl 2: Percutaneous Manipulation of the Fracture}

Spiral or long oblique fracture reductions are amenable to percutaneous clamp placement. The medial tine of the clamp should be kept on the anterior half of the distal femur to avoid injury to the femoral artery in the adductor hiatus. Most distal femur fractures occur distal to this location, making clamp placement relatively safe. If there is concern about clamp proximity to the artery, a $3 \mathrm{~cm}$ split in the quadriceps muscle enables safe clamp placement (e.g. lobster claw or collinear clamp depending on fracture orientation). Joystick manipulation of an anterior to posterior Steinman pin placed out of the planned path of the nail can reduce sagittal plane deformities. Placing the pin on the concave side of coronal plane angulation (lateral for valgus, medial for varus) can correct this deformity concomitantly when flexion and extension is corrected. A percutaneously placed ball spike can be used to correct sagittal malalignment. (Figure 1)

Pearl 3: Blocking Screws

If percutaneous reduction leads to unacceptable reduction after nail placement, blocking screws are the best salvage option barring comminution in the planned screw location. Distal femur fractures typically result in extension and valgus deformities. Therefore, blocking screws lateral to medial in the anterior half and anterior to posterior in the lateral half of the distal segment can improve alignment. Occasionally, varus fractures require blocking screws on the medial side of the nail path. Multiple AP screws on the medial and lateral sides of the nail can add stability to constructs with minimal contact between the nail and distal metaphysis by preventing excessive varus/valgus motion. (Figure 2) 


\section{Pearl 4: Maximal Distal Locking Purchase}

Final nail seating should be assessed with lateral fluoroscopy imaging or direct visualization of the joint surface. The ideal nail position is seated just deep to subchondral bone to maximize screw purchase in the distal segment. Any mechanism inherent to the brand of the nail that can "lock" the interlocking screws to the nail should be utilized. Digital or visual inspection of the knee should be performed after the insertion jig is removed to assure the nail is not prominent.

\section{Pearl 5: Distal Locking and Impacting/Backslapping to Adjust Length}

Distal femur fractures often shorten as the retrograde nail is impacted. Comminuted fractures are particularly prone to shortening, and they lack radiographic reads for determining length. This pitfall is best handled by placing the distal locks in the nail followed by gentle backslapping to restore the proper length to the femur. If cortical landmarks are lacking, a contralateral fluoroscopic ruler measurement from the greater trochanter to the notch can be used as a reference for establishing length on the injured side. Fluoroscopy with a Bovie cord with clamps applied at the trochanter and notch can be used as a substitute.

\section{Pearl 6: Reduction of Articular Fractures Prior to Nailing}

All techniques described above can be utilized in the treatment of intra articular distal femur fractures after articular fractures are reduced and stabilized. This should be done under direct visualization as rotational malreductions of the condyles can be difficult to see with fluoroscopy. Simple sagittal split fractures can be visualized with a small superomedial arthrotomy. Hoffa fractures require a larger arthrotomy placed on the side of the fracture. Lag screws should be placed across articular fractures prior to nailing as clamped fractures tend to 
displace during reaming and nail insertion. Lag screws for sagittal fractures can be easily placed anterior and/or posterior to the planned path of the nail. Anterior to posterior screws for Hoffa fractures can potentially interfere with the critical nail locking screws and may need to be repositioned should this occur. (Figure 3) After fixation of the articular fractures, reaming and nail placement should be done carefully to avoid displacing fragments, and the articular reduction should be rechecked after the nail is fully seated.

Periprosthetic Fractures Around Total Knee Arthroplasty (TKA)

\section{Pearl 1: Starting Point}

Most modern day TKA femoral components have an open box which facilitates retrograde nailing. The starting point is just posterior to the flange and often cannot be assessed until the guide wire is advanced well into the distal fragment. While similar techniques as described for extra articular fractures can be utilized for these fractures, the flange can make manipulation of the fracture more difficult; it will often intussuscept into the canal of the shaft segment. In this circumstance, a bone hook can be placed through a small anterior incision in the quadriceps, and the flange can be pulled out of the canal and maintained in the proper sagittal alignment. (Figure 1) This must be held until the distal locking screws are placed.

\section{Pearl 2: Distal Locking Screws Abutting the Femoral Component}

Similar to Pearl \#4 described above, in periprosthetic fractures, it is important to carefully align the most distal hole in the nail so that the distal interlock rests in very close proximity to the pegs or cement of the femoral component.(Figure 1) This precise distal nail position affords better "purchase" for the distal interlocking screw and improves axial stability. 
There is sparse literature comparing IMN and ORIF in distal femur fractures. Most published studies are small retrospective cases. Additionally, the majority of studies utilized implants that would no longer be considered state-of-the-art. There are a few studies comparing both techniques. Meneghini reported on a series of 95 fractures, 29 treated with IMN and 66 with locked plating. ${ }^{9}$ The nonunion rate was $9 \%$ in the nail group and $19 \%$ in the plate group. Hoskins et al. reported a retrospective review of 297 patients, 195 treated with locked plating and 102 with IMN. ${ }^{10}$ IMN lead to improved quality of life scores at 6 months and slightly less deformity. Beltran described the technical pitfalls and pearls and reviewed results between modern plates and nails. ${ }^{5}$ While no randomized controlled trial between modern nails and plates is yet published, the results of such a trial have been presented by Tornetta et al. on a study of 126 patients. ${ }^{11}$ They reported malalignment in $22 \%$ of IMN cases and $32 \%$ of plate cases. Functional results showed a trend towards better results in the IMN group. Hou et al. reported on fractures adjacent to TKA treated with either IMN or ORIF. ${ }^{12}$ They found no significant differences in fracture alignment and union between the two groups; however, patient numbers were small. They noticed a trend to higher malunion and nonunion rates in the ORIF group.

\section{PROXIMAL TIBIA FRACTURES}

\section{Pearl 1: Semi-extended positioning}

There has been extensive focus on tibial nailing in the semi-extended position in recent years. ${ }^{13-17}$ Maintaining the knee semi-extended rather than flexed helps with fluoroscopic visualization of the fracture and maintenance of reduction. In this position, the starting point can be accessed with a medial or lateral parapatellar approach or with a suprapatellar (or retropatellar) approach. Each approach has its advantages and disadvantages. 
The parapatellar approach can be performed from either the medial or lateral side of the patella depending on surgeon preference and the ease with which the patella subluxates either direction. ${ }^{13} \mathrm{~A}$ full arthrotomy can be performed for better mobilization of the patella and easier access to the proximal tibia starting point. ${ }^{14}$ Alternatively, the surgeon can remain extra articular with a limited fascial incision through the extensor retinaculum while leaving the synovium intact. $^{13}$

Suprapatellar nailing is performed through a longitudinal incision approximately 2 centimeters proximal to the superior pole of the patella. ${ }^{17}$ Access to the proximal tibia is achieved through a specialized cannula that is placed between the patella and trochlea. Clear advantages for the suprapatellar approach are less soft tissue dissection and incisions further from the zone of injury. Disadvantages are possible limited nail diameter due to the size of the cannula and the need for technique-specific instrumentation. The potential for articular cartilage injury has been studied with both suprapatellar and parapatellar nailing. Both approaches have low risk of articular cartilage injury as long as the surgeon is mindful. ${ }^{14,16}$

A technical difference between the suprapatellar approach and parapatellar approaches arises from the need to obtain both an ideal starting point and trajectory. The cannula used for suprapatellar nailing limits posterior translation of the starting guidewire at the level of the trochlea because the guidewire is centered within the cannula. This leads either to the guidewire entry site being forced anteriorly, which may lead to anterior translation of the starting point and increased reaming of the anterior tibial cortex,${ }^{18}$ or to the trajectory being forced posteriorly, which may lead to the nail displacing the fracture. (Figure 4) A simple solution to address this issue is to flex the knee more as this better exposes the starting point. ${ }^{17}$ Knee flexion may be limited due to patellofemoral tightness, but this often can be addressed with a partial 
(superomedial) parapatellar arthrotomy. Alternative techniques to improve suprapatellar guidewire positioning are to perform an anterior drawer maneuver, which results in 3-5mm of physiologic anterior tibial translation and to place the starting wire using only the outer cannula of the telescoping cannula system. Removing the inner cannula before wire placement allows for more freedom with both starting point and trajectory.

\section{Pearl 2: Proper Starting Point}

A good starting point in the proximal tibia is required to prevent fracture malreduction. The proper location on the AP radiograph is just medial to the lateral tibial spine because this point is in line with the intramedullary canal in the sagittal plane. ${ }^{19}$ (Figure 5) Placing the starting point too medial or lateral on the AP view will risk a valgus or varus deformity, respectively. The source of this common mistake is usually assessing the starting point location on a rotationally incorrect AP view. The resultant translation of the starting point is poorly tolerated in proximal fractures. A line drawn inferiorly from the lateral tibial plateau should pass through the center of the fibular head on a properly rotated knee. ${ }^{20}$ (Figure 5)

On the lateral view, the starting point should be located just posterior to the anterior edge of the tibial plateau. (Figure 4) An anterior starting point can lead to excessive reaming of the anterior cortex and also increases the risk of a procurvatum deformity. Once the starting point is established, it is important to maintain its position by avoiding reaming while passing the reamer in or out of the starting hole.

\section{Pearl 3 Adjunctive Reduction Techniques}

The most common deformities resulting from nailing proximal tibia fractures are valgus, procurvatum, and posterior translation of the distal segment. ${ }^{14,20}$ These can be avoided by 
maintaining fracture reduction throughout the entire procedure. Percutaneous clamp application, plates with unicortical screws, and blocking screws as described previously should be used as needed. Percutaneous clamps are particularly effective in the short oblique fractures that often occur in this location. Blocking screws can prevent malreduction of a proximal tibia fracture or restore a reduction that is lost during initial nail passage. Blocking screws are placed where one is trying to prevent the nail from coursing, usually in the proximal segment. ${ }^{21}$ For very proximal fractures, screws in the distal segment may be useful. An anterior-to-posterior screw medial to the intended nail path can prevent valgus, and a medial-to-lateral screw placed posteriorly can prevent procurvatum. (Figure 6)

\section{Pearl 4: Anterior canal trajectory in proximal segment}

Throughout the procedure, the guidewire, reamers, and IMN must maintain an anterior trajectory in the proximal segment. (Figure 4) If the guidewire hits the posterior cortex before the isthmus, the nail will also follow this improper path. As the nail is passed down the intramedullary canal, it will deflect off the posterior cortex and force the proximal segment into procurvatum as it passes into the isthmus. Medial-to-lateral blocking screws in the metaphysis just posterior to the anticipated path of the nail can prevent a posterior trajectory of the guidewire. (Figure 6) If the guidewire persistently passes posterior even after placement of blocking screws (e.g. due to the patella, femur, or soft tissue limiting repositioning), the wire can be advanced just proximal to the blocking screw. A medial unicortical Steinmann pin directed just posterior to the guidewire can be placed percutaneously. The guidewire can be manipulated anteriorly by the Steinmann pin within the intramedullary canal by pushing the external portion of the pin posteriorly, using intact medial cortex as a fulcrum. Once the guidewire position is corrected, it can be advanced anterior to the blocking screws. 


\section{Pearl 5: Posterior supplemental fixation for intra articular fractures}

All of the techniques described above apply to intra articular proximal tibia fracture nailing. The difference in these cases is that the articular block must be reconstructed prior to IMN. To achieve this, standard reduction and fixation techniques for tibial plateau fractures are utilized. ${ }^{8}$ However, the surgeon must be mindful to avoid placement of screws in eventual path of the nail. This means placing proximal screws posteriorly. (Figure 7)

Simple medial or lateral articular splits that do not communicate with separate extra articular fractures can be fixed with buttress plates or screws alone. ${ }^{8}$ If the split communicates with the metaphyseal or diaphyseal component of the fracture, screws alone can be used to fix the proximal segment before IMN, but this may lead to difficulty maintaining length and alignment of the metaphyseal portion of the fracture. Provisional fixation with a bridge plate or multiple smaller plates can be used prior to nailing. ${ }^{7}$ (Figure 7)

Proximal Tibia Literature

IMN is a well-established method for treating extra articular proximal tibia fractures. ${ }^{14,22,23}$ Ryan et al. retrospectively reviewed semi-extended nailing (partial medial parapatellar arthrotomy) of proximal and distal metaphyseal fractures versus "standard nailing" with the knee in flexion. ${ }^{14}$ At the time of fracture union, they found no significant difference in the incidence of knee pain between the two groups or quality of reduction. It is not clear whether there are any clinically significant advantages to the suprapatellar versus the parapatellar approach.

Meena et al randomized 58 patients with proximal metaphyseal tibia fractures to lateral percutaneous locked plating versus intramedullary nail fixation. ${ }^{24}$ The intramedullary nail group 
had a significantly shorter average hospital stay (4.1 days vs. 5.3 days) and time to fracture union (18.26 vs. 22.84 weeks). Complications were similar between groups.

Yoon et al. examined proximal tibia fractures treated with a combination of plate and screw fixation followed by intramedullary nail stabilization in a case series. ${ }^{7}$ Twenty-five of twenty-seven fractures achieved union, and there were no cases of late fracture displacement.

\section{DISTAL TIBIA FRACTURES}

\section{Pearl 1: Obtaining and maintaining reduction}

Valgus deformity is the most common malalignment encountered during nailing of distal tibia fractures, followed by recurvatum and varus. ${ }^{25-27}$ Reports of high malunion rates demonstrate intraoperative malreductions rather than late displacement. ${ }^{25,26,28}$ Previously described reduction techniques should be used as needed. The triangular cross-section of the tibia often leads to percutaneously placed clamps slipping during fracture manipulation. Drilling precisely placed unicortical holes in which to seat clamp tines on the medial and lateral surfaces of the tibia resolves this issue. (Figure 8) Unicortical plates/screws may facilitate reduction and provide temporary stabilization.

\section{Pearl 2: Central nail position in metaphyseal segment}

Precise nail placement in the distal segment is critical to avoid deformity. The center of the nail on the AP view should rest in line with the tibia's anatomic axis, which intersects the talar dome between the center and up to ten millimeters lateral. Appropriately placed blocking screws to create a "neo-cortex" and centralize the nail within the metaphysis are useful in 
preventing malalignment. ${ }^{21} \mathrm{An}$ anterior to posterior directed blocking screw in the medial onethird of the distal tibia prevents medial nail positioning and subsequent valgus deformity. (Figure 8)

Pearl 3: Maximal use of interlocking screws

Fracture morphology should allow at least two interlocking screws to be placed in the distal segment. ${ }^{29}$ When possible, three interlocking screws should be inserted distally with at least one screw out of plane from the others. ${ }^{30}$ (Figure 9) Use of only one distal interlocking screw is associated with higher rates of early implant failure and nonunion. ${ }^{31}$

\section{Pear 4: Fibula fixation}

Fixation of the fibula has been shown in some series to facilitate reduction, improve alignment, and prevent late displacement. ${ }^{25,26,32}$ This can be achieved with open reduction and plate fixation or closed versus percutaneous clamp reduction and retrograde intramedullary small fragment screw fixation. (Figures 8 and 9)

Improvement of tibia reduction with fibular fixation is not an absolute necessity, and there may be injury, surgeon, or patient factors that affect this relationship. Researchers found a low malunion rate (3\%) in a consecutive series of 122 patients with distal tibia fractures without fibular fixation. ${ }^{29}$ Furthermore, there is concern for increased risk of distal tibial nonunion after fibular stabilization. ${ }^{26}$

\section{Pearl 5: Semi-extended positioning}

Although originally described for proximal tibial nailing, semi-extended positioning of the leg during IMN improves clinical and fluoroscopic evaluation of the distal tibia, leading to 
low rates of malalignment. ${ }^{14,33}$ Various semi-extended approaches are covered in detail in the proximal tibia section above.

\section{Pearl 6: Independent fixation of intra articular fractures}

Identification and anatomic reduction of intra articular fractures should precede intramedullary fixation. (Figure 9) CT scanning is recommended for all fractures extending to the distal tibia metaphysis to delineate pilon fracture planes and determine optimal screw placement for fixation of the articular block out of the future nail pathway. Displaced posterior malleolar fractures can be reduced followed by stabilization with independent small fragment screw(s) or an anterior-to-posterior interlocking screw through the nail. There is no evidence to support routine fixation of small, nondisplaced posterior malleolar fragments.

\section{Distal Tibia Literature}

Meta-analyses and systematic reviews comparing plate and nail fixation of distal tibia fractures have yielded limited information to guide treatment. ${ }^{34-36}$ Two RCTs found no difference in functional outcome between plates and nails for distal tibia fractures. ${ }^{28,37}$ Two RCTs demonstrated a lower rate of wound complications and superficial infection with IMN. ${ }^{28,38}$ Three RCTs reported no differences in infection and wound healing. ${ }^{25,37,39}$ The most significant discrepancy in the literature is the rate of primary (intraoperative) malalignment. The largest RCT and several retrospective studies reported the incidence of malalignment following IMN between $20 \%$ and $30 \%$ in comparison to less than $10 \%$ with plate fixation. ${ }^{25,26,28}$ These nails were all placed with an infrapatellar approach. 
In contrast, the semi-extended IMN technique has shown much more favorable

results. ${ }^{14,29,33}$ A recent retrospective review by Avilucea, et al. demonstrated a 3.8\% rate of malalignment in distal tibia fractures treated with a suprapatellar approach compared to a $26.1 \%$ rate of malalignment in those treated with an infrapatellar approach. ${ }^{33}$ However, Barcak, et al. found a low rate of malalignment $(3 \%)$ in patients with distal tibia fractures within $5 \mathrm{~cm}$ of the joint treated by infrapatellar nailing. ${ }^{40}$

\section{Conclusion}

PIMN of tibia and distal femur fractures is a well-established treatment yielding excellent outcomes. No studies have demonstrated that IMN compared to plating predisposes periarticular fractures to loss of reduction during the postoperative period, but many have reported on residual intraoperative malalignment. ${ }^{4,14,25,26,29,33,40}$ Therefore, success with extreme nailing hinges on the planning and execution of intraoperative strategies to effect an acceptable reduction.

\section{$\underline{\text { References }}$}

1. Mehling I, Hoehle P, Sternstein W, Blum J, Rommens PM. Nailing versus plating for comminuted fractures of the distal femur: a comparative biomechanical in vitro study of three implants. Eur J Trauma Emerg Surg. 2013;39(2):139-146.

2. Casstevens C, Le T, Archdeacon MT, Wyrick JD. Management of extra-articular fractures of the distal tibia: intramedullary nailing versus plate fixation. J Am Acad Orthop Surg. 2012;20(11):675-683.

3. Probe R. Semiextending Nailing for Combined Shaft and Ankle Injuries of the Leg. $J$ Orthop Trauma. 2016;30 Suppl 2:S37-38.

4. Nork SE, Schwartz AK, Agel J, Holt SK, Schrick JL, Winquist RA. Intramedullary nailing of distal metaphyseal tibial fractures. J Bone Joint Surg Am. 2005;87(6):1213-1221.

5. Beltran MJ, Gary JL, Collinge CA. Management of distal femur fractures with modern plates and nails: state of the art. J Orthop Trauma. 2015;29(4):165-172.

6. Krettek C, Stephan C, Schandelmaier P, Richter M, Pape HC, Miclau T. The use of Poller screws as blocking screws in stabilising tibial fractures treated with small diameter intramedullary nails. $J$ Bone Joint Surg Br. 1999;81(6):963-968.

7. Yoon RS, Bible J, Marcus MS, et al. Outcomes following combined intramedullary nail and plate fixation for complex tibia fractures: A multi-centre study. Injury. 2015;46(6):1097-1101. 
8. Kubiak EN, Camuso MR, Barei DP, Nork SE. Operative treatment of ipsilateral noncontiguous unicondylar tibial plateau and shaft fractures: combining plates and nails. J Orthop Trauma. 2008;22(8):560-565.

9. Meneghini RM, Keyes BJ, Reddy KK, Maar DC. Modern retrograde intramedullary nails versus periarticular locked plates for supracondylar femur fractures after total knee arthroplasty. $J$ Arthroplasty. 2014;29(7):1478-1481.

10. Hoskins W, Sheehy R, Edwards ER, et al. Nails or plates for fracture of the distal femur? data from the Victoria Orthopaedic Trauma Outcomes Registry. Bone Joint J. 2016;98-B(6):846-850.

11. Tornetta $\mathrm{P}$, III, Egol KA, Jones $\mathrm{CB}$, et al. Locked Plating Versus Retrograde Nailing for Distal Femur Fractures: A Multicenter Randomized Trial. Paper presented at: Orthopaedic Trauma Association Annual Meeting2013; Phoenix, Arizona.

12. Hou Z, Bowen TR, Irgit K, et al. Locked plating of periprosthetic femur fractures above total knee arthroplasty. J Orthop Trauma. 2012;26(7):427-432.

13. Kubiak EN, Widmer BJ, Horwitz DS. Extra-articular technique for semiextended tibial nailing. J Orthop Trauma. 2010;24(11):704-708.

14. Ryan SP, Steen B, Tornetta P, 3rd. Semi-extended nailing of metaphyseal tibia fractures: alignment and incidence of postoperative knee pain. J Orthop Trauma. 2014;28(5):263-269.

15. Gelbke MK, Coombs D, Powell S, DiPasquale TG. Suprapatellar versus infra-patellar intramedullary nail insertion of the tibia: a cadaveric model for comparison of patellofemoral contact pressures and forces. J Orthop Trauma. 2010;24(11):665-671.

16. Sanders RW, DiPasquale TG, Jordan CJ, Arrington JA, Sagi HC. Semiextended intramedullary nailing of the tibia using a suprapatellar approach: radiographic results and clinical outcomes at a minimum of 12 months follow-up. J Orthop Trauma. 2014;28(5):245-255.

17. Eastman J, Tseng S, Lo E, Li CS, Yoo B, Lee M. Retropatellar technique for intramedullary nailing of proximal tibia fractures: a cadaveric assessment. J Orthop Trauma. 2010;24(11):672-676.

18. Bible JE, Choxi AA, Dhulipala S, Evans JM, Mir HR. Quantification of anterior cortical bone removal and intermeniscal ligament damage at the tibial nail entry zone using parapatellar and retropatellar approaches. J Orthop Trauma. 2013;27(8):437-441.

19. Tornetta P, 3rd, Riina J, Geller J, Purban W. Intraarticular anatomic risks of tibial nailing. J Orthop Trauma. 1999;13(4):247-251.

20. Walker RM, Zdero R, McKee MD, Waddell JP, Schemitsch EH. Ideal tibial intramedullary nail insertion point varies with tibial rotation. J Orthop Trauma. 2011;25(12):726-730.

21. Krettek C, Miclau T, Schandelmaier P, Stephan C, Mohlmann U, Tscherne H. The mechanical effect of blocking screws ("Poller screws") in stabilizing tibia fractures with short proximal or distal fragments after insertion of small-diameter intramedullary nails. J Orthop Trauma. 1999;13(8):550-553.

22. Nork SE, Barei DP, Schildhauer TA, et al. Intramedullary nailing of proximal quarter tibial fractures. J Orthop Trauma. 2006;20(8):523-528.

23. Lindvall E, Sanders R, Dipasquale T, Herscovici D, Haidukewych G, Sagi C. Intramedullary nailing versus percutaneous locked plating of extra-articular proximal tibial fractures: comparison of 56 cases. J Orthop Trauma. 2009;23(7):485-492.

24. Meena RC, Meena UK, Gupta GL, Gahlot N, Gaba S. Intramedullary nailing versus proximal plating in the management of closed extra-articular proximal tibial fracture: a randomized controlled trial. J Orthop Traumatol. 2015;16(3):203-208.

25. Vallier HA, Cureton BA, Patterson BM. Randomized, prospective comparison of plate versus intramedullary nail fixation for distal tibia shaft fractures. J Orthop Trauma. 2011;25(12):736741. 
26. Vallier HA, Le TT, Bedi A. Radiographic and clinical comparisons of distal tibia shaft fractures (4 to $11 \mathrm{~cm}$ proximal to the plafond): plating versus intramedullary nailing. J Orthop Trauma. 2008;22(5):307-311.

27. Robinson CM, McLauchlan GJ, McLean IP, Court-Brown CM. Distal metaphyseal fractures of the tibia with minimal involvement of the ankle. Classification and treatment by locked intramedullary nailing. J Bone Joint Surg Br. 1995;77(5):781-787.

28. Im GI, Tae SK. Distal metaphyseal fractures of tibia: a prospective randomized trial of closed reduction and intramedullary nail versus open reduction and plate and screws fixation. $J$ Trauma. 2005;59(5):1219-1223; discussion 1223.

29. De Giacomo AF, Tornetta P, 3rd. Alignment After Intramedullary Nailing of Distal Tibia Fractures Without Fibula Fixation. J Orthop Trauma. 2016;30(10):561-567.

30. Chan DS, Nayak AN, Blaisdell G, et al. Effect of distal interlocking screw number and position after intramedullary nailing of distal tibial fractures: a biomechanical study simulating immediate weight-bearing. J Orthop Trauma. 2015;29(2):98-104.

31. Mohammed A, Saravanan R, Zammit J, King R. Intramedullary tibial nailing in distal third tibial fractures: distal locking screws and fracture non-union. Int Orthop. 2008;32(4):547-549.

32. Egol KA, Weisz R, Hiebert R, Tejwani NC, Koval KJ, Sanders RW. Does fibular plating improve alignment after intramedullary nailing of distal metaphyseal tibia fractures? J Orthop Trauma. 2006;20(2):94-103.

33. Avilucea FR, Triantafillou K, Whiting PS, Perez EA, Mir HR. Suprapatellar Intramedullary Nail Technique Lowers Rate of Malalignment of Distal Tibia Fractures. J Orthop Trauma. 2016;30(10):557-560.

34. Zelle BA, Bhandari M, Espiritu M, Koval KJ, Zlowodzki M, Evidence-Based Orthopaedic Trauma Working G. Treatment of distal tibia fractures without articular involvement: a systematic review of 1125 fractures. J Orthop Trauma. 2006;20(1):76-79.

35. Iqbal HJ, Pidikiti P. Treatment of distal tibia metaphyseal fractures; plating versus intramedullary nailing: a systematic review of recent evidence. Foot Ankle Surg. 2013;19(3):143-147.

36. Mao Z, Wang G, Zhang L, et al. Intramedullary nailing versus plating for distal tibia fractures without articular involvement: a meta-analysis. J Orthop Surg Res. 2015;10:95.

37. Mauffrey C, McGuinness K, Parsons N, Achten J, Costa ML. A randomised pilot trial of "locking plate" fixation versus intramedullary nailing for extra-articular fractures of the distal tibia. $J$ Bone Joint Surg Br. 2012;94(5):704-708.

38. Guo JJ, Tang N, Yang HL, Tang TS. A prospective, randomised trial comparing closed intramedullary nailing with percutaneous plating in the treatment of distal metaphyseal fractures of the tibia. J Bone Joint Surg Br. 2010;92(7):984-988.

39. Li Y, Jiang X, Guo Q, Zhu L, Ye T, Chen A. Treatment of distal tibial shaft fractures by three different surgical methods: a randomized, prospective study. Int Orthop. 2014;38(6):1261-1267.

40. Barcak E, Collinge CA. Metaphyseal Distal Tibia Fractures: A Cohort, Single-Surgeon Study Comparing Outcomes of Patients Treated With Minimally Invasive Plating Versus Intramedullary Nailing. J Orthop Trauma. 2016;30(5):e169-174. 